\title{
ON THE PLASTIC BENDING OF CIRCULAR PLATES UNDER UNIFORM TRANSVERSE LOADS*
}

\author{
BY \\ D. TRIFAN \\ Brown University
}

1. Introduction. The primary purpose of this paper is to investigate the mechanical behaviour of thin plates subjected to distributed transverse loads which produce plastic deformations. Throughout this discussion the plates will be considered as being homogeneous and composed of isotropic materials exhibiting a gradual transition from the elastic to the plastic state. Two alternative theories of plasticity, namely a theory of plastic flow and a theory of plastic deformation, ${ }^{1}$ will be used, and their predictions will be compared with each other. In particular, the case of a circular plate subjected to a uniformly distributed transverse load will be considered in detail.

As applied to strain-hardening materials, the theory of plastic flow is based on the assumption that the rate of change of strain is uniquely determined by the existing stress and the rate of change of stress. Stress-strain relations of this type have been considered by Prager [2, 3] and, more recently, by Handelman, Lin, and Prager [4]; they may be considered as a natural generalization of the stress-strain relations proposed for perfectly plastic materials by Prandtl [5] and Reuss [6]. On the other hand the theory of plastic deformation developed by Hencky [7] for perfectly plastic materials and applied by Nadai [8, p. 75] to materials with continuous transition from the elastic to the plastic state is based on the assumption that in the plastic state as in the elastic state, the state of strain is uniquely determined by the state of stress. These stressstrain relations [9] are simpler than those of the flow theory, and thus have received more consideration in mathematical studies concerning permanent deformations in structural elements.

Until recently, comparatively little attention had been given to the plastic bending of plates; however, some notable contributions in this field have recently been made by Russian investigators, in particular, Ilyushin [10] and Sokolovsky [11]. Most of their results, however, do not apply to materials which exhibit gradual transition from the elastic to the plastic state.

2. Stress-strain relations. Investigation of the bending of plates made of a strainhardening material by means of the theory of plastic flow is extremely difficult if the customary form of the stress-strain relations for such materials is adopted [4, Eq. 27]. Indeed, from this form of the stress-strain relations, the rate of change of the stress tensor can not be obtained explicitly in terms of the rate of change of the strain tensor, the strain tensor itself, and the strain invariants, as is necessary in the study of the bending of plates. This difficulty is avoided if slightly modified stress-strain relations for loading are adopted. Let the tensors of stress and strain be denoted by $\rho_{i j}$ and $\epsilon_{i j}$, their deviations by

*Received Jan. 30, 1948. The conclusions presented in this paper were obtained in the course of research conducted under Contract N7onr-358 sponsored jointly by the Office of Naval Research and the Bureau of Ships.

1This terminology is due to A. A. Ilyushin [1] (numbers in square brackets refer to the bibliography at the end of the paper). 


$$
r_{i i}=\rho_{i i}-\frac{1}{3} \rho_{k k} \delta_{i j}, \quad e_{i i}=\epsilon_{i i}-\frac{1}{3} \epsilon_{k k} \delta_{i j}
$$

$\left(\delta_{i j}=\right.$ Kronecker delta $)$, and the elastic shear modulus and the tangent shear modulus by $G_{0}$ and $G$, respectively, the latter being assumed as a function of the strain intensity

$$
E=\frac{2}{3} e_{i j} e_{i j} \text {. }
$$

The stress-strain relation of the flow theory used in this paper then has the form

$$
r_{i j}^{*}=2 G_{0} e_{i j}^{*}-\left(G_{0}-G\right) \frac{E^{*}}{E} e_{i j} .
$$

Throughout the following discussion, the Latin subscripts run from 1 through 3 , and rates of change of variables with respect to time will be indicated by asterisks. Stressstrain relations of the type (2.1) may be termed stress theories of plastic flow, while relations such as are treated in [4] may be called strain theories of plastic flow.

The expression (2.1) constitutes five linearly independent stress-strain relations which take into account only the change of shape of a deformed body. To these, a sixth expression dealing with the change in volume must be added. For the purpose of simplification, we shall assume the material to be incompressible:

$$
\epsilon_{k k}=0 .
$$

(Here, repeated indices indicate summation in accordance with the customary summation convention of tensor calculus.) Accordingly, Poisson's ratio $\nu=1 / 2, \epsilon_{i}, \equiv e_{i i}$, and Young's modulus equals three times the shear modulus $G_{0}$.

In order to determine the quantity $G$ which is a function of the strain intensity $E$ only and thus independent of the specific type of loading, it is sufficient to consider a simple state of stress, e.g., simple tension. Indicating the tensile stress by $\rho$ and the corresponding unit extension by $\epsilon$, Eq. (2.1) reduces to the single equation

$$
\frac{d \rho}{d \epsilon}=3 G,
$$

and the strain intensity becomes $E=\epsilon^{2}$. Hence the tangent shear modulus $G$ for any material obeying (2.1) can be determined if the stress-strain diagram for simple tension is known. For the material being considered here, the function $\rho=\rho(\epsilon)$ can be represented with sufficient accuracy by the power series,

$$
\rho=a_{1} \epsilon+a_{3} \epsilon^{3}+a_{5} \epsilon^{5}+\cdots,
$$

where $a_{1}, a_{3}, a_{5}$, etc. are constants. Thus the tangent shear modulus is given by

$$
3 G=a_{1}+3 a_{3} E+5 a_{5} E^{2}+\cdots,
$$

where $a_{1}$ equals Young's modulus, i.e., $a_{1}=3 G_{0}$.

It is more convenient from this point on to work with non-dimensional quantities. Accordingly, the following non-dimensional components of stress and stress deviation will be introduced: $\sigma_{i j}=\rho_{i j} / G_{0}, s_{i j}=r_{i j} / G_{0}$. By substituting these quantities and the series expansion (2.5) into Eq. (2.1), we obtain a stress-strain relation for the stress theory of plastic flow for a material with a stress-strain curve in simple tension which is represented by the series (2.4), or by the equivalent general stress-strain relation 


$$
s_{i i}^{*}=2 e_{i j}^{*}-\left[b_{1}+b_{2} E+b_{3} E^{2}+\cdots\right] E^{*} e_{i j},
$$

where

$$
b_{n}=-(2 n+1) a_{2 n+1} / 3 G_{0}, \quad n=1,2,3, \cdots
$$

The stress-strain relation of the theory of plastic deformation is of the form

$$
r_{i j}=2 G e_{i i},
$$

where $G^{\prime}=G^{\prime}(E)$ is the secant shear modulus. For simple tension Eq. (2.7) reduces to the single expression

$$
\rho / \epsilon=3 G .
$$

Using the same series expansion (2.4) as in the plastic flow theory, we obtain

$$
3 G^{n}=a_{1}+a_{3} E+a_{5} E^{2}+\cdots
$$

Substituting this into Eq. (2.7) and using the non-dimensional form, we find

where

$$
s_{i j}=2 e_{i},-\left[c_{1}+c_{2} E+c_{3} E^{2}+\cdots\right] E e_{i j},
$$

$$
c_{n}=-2 a_{2 n+1} / 3 G_{0} \text {. }
$$

3. Basic differential equations. We consider a thin plate subjected to distributed transverse loads, and assume that the resulting deflections are small in comparison to the thickness of the plate. We introduce the rectangular coordinates $X_{i}$, the $X_{1}, X_{2}$ plane coinciding with the plane horizontal middle surface of the undeformed plate, and the axis of $X_{3}$ being directed vertically downward. As is usual in the theory of thin plates, the stress components $\sigma_{13}, \sigma_{23}, \sigma_{33}$ will be treated as small in comparison with the stresses parallel to the middle surface of the plate $\left(\sigma_{11}, \sigma_{22}\right.$ and $\left.\sigma_{12}\right)$. Restricting Greek subscripts to values 1 and 2, we may, therefore, write Eq. (2.6) in the form

$$
\sigma_{\alpha \beta}^{*}=2\left\{e_{\alpha \beta}^{*}+\delta_{\alpha \beta} e_{\gamma \gamma}^{*}\right\}-\left\{e_{\alpha \beta}+\delta_{\alpha \beta} e_{\gamma \gamma}\right\}\left[b_{1}+b_{2} E+\cdots\right] E^{*},
$$

where $E=2 / 3\left(e_{\alpha \beta} e_{\alpha \beta}+e_{\alpha \alpha} e_{\beta \beta}\right)$.

We now adopt the following notations:

$$
\begin{gathered}
x_{\alpha}=X_{\alpha} / R_{0}, \quad x_{3}=2 X_{3} / h, \\
w=W h / 2 R_{0}^{2}, \quad M_{\alpha \beta}=4 \mathbb{M}_{\alpha \beta} / h^{2} G_{0}, \\
l=4 q R_{0}^{2} / h^{2} G_{0},
\end{gathered}
$$

where $R_{0}$ is a fixed suitably chosen length, $h$ the constant thickness of the plate, $W=$ $W\left(X_{\alpha}\right)$ the deflection, $q$ the intensity of the load, and $\mathfrak{M}_{\alpha \beta}$ are the flexural and twisting moments $\left(\mathfrak{M}_{\alpha \beta}=\int_{-h / 2}^{h / 2} X_{3} \rho_{\alpha \beta} d X_{3}\right)$.

It is a fundamental hypothesis in the theory of elastic plates that points on a normal to the middle surface of the plate before deformation remain on a normal to the deformed middle surface. It is assumed that this hypothesis remains valid for the plastic materials considered here. Accordingly, the relation between the strains $\epsilon_{\alpha \beta}$ and the deflection $W$ of the middle surface is $[12, \mathrm{p} .323]$

$$
\epsilon_{\alpha \beta}=-X_{3} W_{, \alpha \beta},
$$

where $W_{, \alpha \beta}=\partial^{2} W / \partial X_{\alpha} \partial X_{\beta}$. 
In non-dimensional form this expression becomes

$$
\epsilon_{\alpha \beta}=-x_{3} w_{, \alpha \beta} \text {. }
$$

In terms of the deflection $w$, Eq. (3.1) then becomes

$$
\sigma_{\alpha \beta}^{*}=-2 x_{3} \Omega_{\alpha \beta}^{*}+\frac{2}{3} b_{1} x_{3}^{3} E_{0}^{*} \Omega_{\alpha \beta}+\frac{4}{9} b_{2} x_{3}^{5} E_{0} E_{0}^{*} \Omega_{\alpha \beta}+\cdots,
$$

where the quantity $E_{0}$, related to $E$ by means of $E=2 / 3 x_{3}^{2} E_{0}$, is written as

$$
\begin{gathered}
E_{0}=\Omega_{\alpha \beta} w_{, \alpha \beta}, \\
\Omega_{\alpha \beta}=w_{, \alpha \beta}+\delta_{\alpha \beta} w_{, \gamma \gamma} .
\end{gathered}
$$

The time rate of change of the non-dimensional bending moments

$$
M_{\alpha \beta}^{*}=\int_{-1}^{1} x_{3} \sigma_{\alpha \beta}^{*} d x_{3}
$$

can now be easily calculated. We obtain

$$
M_{\alpha \beta}^{*}=-\frac{4}{3}\left[\Omega_{\alpha \beta}^{*}-\frac{1}{5} b_{1} E_{0}^{*} \Omega_{\alpha \beta}-\frac{2}{21} b_{2} E_{0}^{*} E_{0} \Omega_{\alpha \beta}-\cdots\right] .
$$

For transversely loaded plates with small deflections, the equation of equilibrium ${ }^{2}$ $[13$, p. 87] becomes

$$
M_{\alpha \beta, \alpha \beta}^{*}+l^{*}=0
$$

which, together with Eq. (3.6), furnishes

$$
\Omega_{\alpha \beta, \alpha \beta}^{*}-\frac{1}{5} b_{1}\left\{E_{0}^{*} \Omega_{\alpha \beta}\right\}_{, \alpha \beta}-\frac{2}{21} b_{2}\left\{E_{0}^{*} E_{0} \Omega_{\alpha \beta}\right\}_{, \alpha \beta}-\cdots=\frac{3}{4} l^{*} .
$$

Finally, referring to definitions (3.5) and carrying out the necessary steps, we arrive at the basic non-linear partial differential equation in $w$ for the flow theory, namely

$$
\begin{aligned}
w_{, \alpha \alpha \beta \beta}^{*} & -\frac{b_{1}}{5}\left\{w_{, \alpha \beta} w_{, \gamma \delta} w_{, \alpha \beta \gamma \delta}^{*}+2 w_{, \alpha \alpha} w_{, \gamma \delta} w_{, \beta \beta \gamma \delta}^{*}+w_{, \alpha \alpha} w_{, \beta \beta} w_{, \gamma \gamma \delta \delta}^{*}\right. \\
+ & 2 w_{, \alpha \beta} w_{, \alpha \gamma \delta} w_{, \beta \gamma \delta}^{*}+2 w_{, \alpha \alpha} w_{, \beta \gamma \delta} w_{, \beta \gamma \delta}^{*}+2 w_{, \alpha \beta} w_{, \alpha \gamma \gamma} w_{, \beta \delta \delta}^{*} \\
& +6 w_{, \alpha \alpha} w_{, \beta \gamma \gamma} w_{, \beta \delta \delta}^{*}+4 w_{, \alpha \beta} w_{, \gamma \gamma \delta} w_{, \alpha \beta \delta}^{*}+w_{, \alpha \beta} w_{, \alpha \beta \gamma \delta} w_{, \gamma \delta}^{*} \\
& +w_{, \alpha \alpha} w_{, \beta \beta \gamma \delta} w_{, \gamma \delta}^{*}+w_{, \alpha \beta} w_{, \alpha \beta \gamma \gamma} w_{, \delta \delta}^{*}+3 w_{, \alpha \alpha} w_{, \beta \beta \gamma \gamma} w_{, \delta \delta}^{*} \\
& \left.+4 w_{, \alpha \beta \beta} w_{, \alpha \gamma \delta} w_{, \gamma \delta}^{*}+4 w_{\alpha \beta \beta} w_{, \alpha \gamma \gamma} w_{, \delta \delta}^{*}+2 w_{, \alpha \beta} w_{, \gamma \gamma \delta \delta} w_{, \alpha \beta}^{*}\right\} \\
& -\frac{b_{2}}{21}\left\{E_{0}^{*} E_{0} \Omega_{\alpha \beta}\right\}_{, \alpha \beta}-\cdots=\frac{3}{8} l^{*} .
\end{aligned}
$$

The corresponding basic partial differential equation for the plastic deformation theory can be obtained in a similar manner. We note merely the final equation,

${ }^{2}$ See Eq. (g): $\mathfrak{M}_{11}=M_{x}, \mathfrak{M}_{22}=M_{y}, \mathfrak{P}_{12}=-M_{x y}, \mathfrak{R}_{21}=M_{y z}$. 


$$
\begin{gathered}
w_{, \alpha \alpha \beta \beta}-\frac{c_{1}}{5}\left\{w_{, \alpha \beta} w_{, \gamma \delta} w_{, \alpha \beta \gamma \delta}+2 w_{, \alpha \alpha} w_{, \beta \gamma} w_{, \beta \gamma \delta \delta}+2 w_{, \alpha \alpha} w_{, \beta \beta} w_{, \gamma \gamma \delta \delta}\right. \\
+w_{, \alpha \beta} w_{, \alpha \beta} w_{, \gamma \gamma \delta \delta}+w_{, \alpha \beta} w_{, \alpha \gamma \delta} w_{, \beta \gamma \delta}+w_{, \alpha \beta} w_{, \alpha \gamma \gamma} w_{, \beta \delta \delta} \\
\left.+4 w_{, \alpha \beta} w_{, \alpha \beta \gamma} w_{, \gamma \delta \delta}+w_{, \alpha \alpha} w_{, \beta \gamma \delta} w_{, \beta \gamma \delta}+5 w_{, \alpha \alpha} w_{, \beta \gamma \gamma} w_{, \beta \delta \delta}\right\} \\
-\frac{c_{2}}{21}\left\{E_{0}^{*} E_{0} \Omega_{\alpha \beta}\right\}_{, \alpha \beta}-\cdots=\frac{3}{8} l .
\end{gathered}
$$

4. Example. We are now in a position to consider a specific problem in detail, namely the case where

a) the thin plate is made of $24 \mathrm{~S}-\mathrm{T}$ aluminum alloy,

b) the contour of the plate is circular,

c) the plate is built-in along its entire boundary, and

d) the lateral load is uniformly distributed.

In Fig. 1 the graph (full curve) of the stress $\rho$ and the corresponding unit extension $\epsilon$ is given for a tension test with a specimen of $24 \mathrm{~S}-\mathrm{T}$ aluminum. The analytic approximation for this curve most suitable for our purposes is

$$
\rho=3 G_{0}\left(\epsilon-\frac{10^{6}}{128} \epsilon^{3}\right)
$$

where

$$
3 G_{0}=10.667 \times 10^{6} \mathrm{lbs} . / \mathrm{sq} . \text { in. }
$$

This relation is represented by the dashed curve in Fig. 1. Referring to the expansion (2.4), we find that the values of the coefficients for this material become

$$
a_{1}=3 G_{0}, \quad a_{3}=-\frac{3 \cdot 10^{6}}{128} G_{0}, \quad a_{5}=a_{7}=\cdots=0 .
$$

From this it follows (see Eqs. (2.6) and (2.9)) that

$$
b_{1}=\frac{3 \cdot 10^{6}}{128}, \quad b_{2}=b_{3}=\cdots=0
$$

and

$$
c_{1}=\frac{2 \cdot 10^{6}}{128}, \quad c_{2}=c_{3}=\cdots=0 .
$$

With these values, the basic equations (3.9) and (3.10) are greatly simplified.

Since the load intensity is assumed to be constant, i.e., $l \equiv l_{0}$, the deflection $w$ is independent of the polar angle $\theta$. The transformation of Eq. (3.9) with the values (4.2) into the more suitable polar coordinate system gives a differential equation in $w$, now a function of the single non-dimensional radial coordinate $r=R / R_{0}$, where $R$ is the dimensional radial coordinate and $R_{0}$ the outer radius. The actual transformation is quite laborious but straightforward, and for that reason it will be omitted here. Expressing the differentiation with respect to $r$ by primes, we find

$$
w^{* \mathrm{iv}}+\frac{2}{r} w^{* \prime \prime \prime}-\frac{1}{r^{2}} w^{* \prime \prime}+\frac{1}{r^{3}} w^{* \prime}-\frac{b_{1}}{5} F=\frac{3}{8} l_{0}^{*},
$$


where $F=F\left(r, t, w^{\prime}, w^{\prime \prime}, w^{\prime \prime \prime}, w^{\mathrm{iv}}, w^{* \prime}, w^{* \prime \prime}, w^{* \prime \prime \prime}, w^{* \mathrm{iv}}\right)$ represents 24 terms and $l_{0}=$ $l_{0}(t)$.

The complete representation of Eq. (4.3) shows that this expression is homogeneous in the time rates of change, and therefore the time variable $t$ can be changed to any

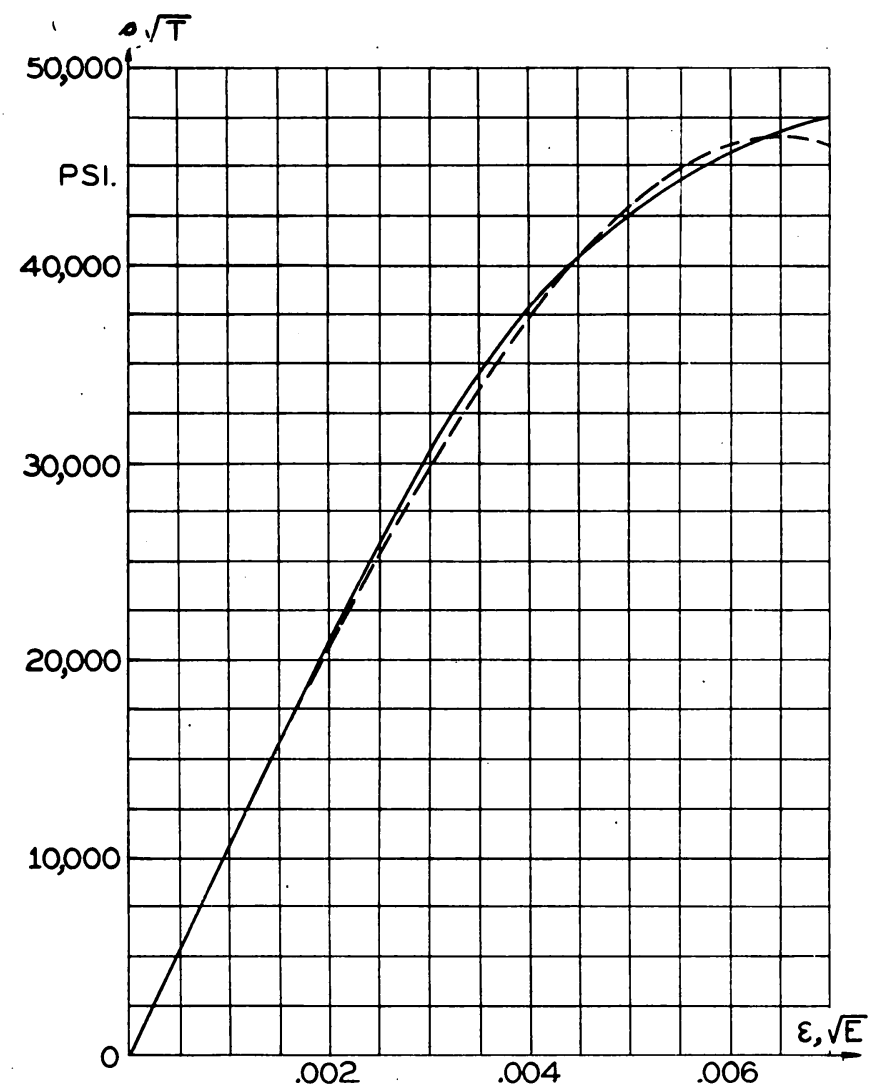

FIg. 1. Stress-strain diagrams: (1) 24S-T Aluminum Alloy (full curve); (2) Approximation as given by Eq. (4.1) (dashed curve).

other monotonically increasing variable. Since we assume the load to increase with time, $t$ can be replaced by $l_{0}$, thus, $w^{*}=l_{0}^{*}\left(d w / d l_{0}\right)$.

It is found that if Eq. (4.3) is multiplied by the factor $r$ then the differential equation can be integrated to a lower order expression, namely

$$
\begin{aligned}
& r w^{* \prime \prime \prime}+w^{* \prime \prime}-\frac{1}{r} w^{* \prime}-\frac{b_{1}}{5}\left\{\left(4 r w^{\prime \prime} w^{\prime \prime}+4 w^{\prime} w^{\prime \prime}+\frac{1}{r} w^{\prime} w^{\prime}\right) w^{* \prime \prime \prime}\right. \\
& +\left(8 r w^{\prime \prime} w^{\prime \prime \prime}+8 w^{\prime \prime} w^{\prime \prime}+4 w^{\prime} w^{\prime \prime \prime}+\frac{2}{r} w^{\prime} w^{\prime \prime}-\frac{1}{r^{2}} w^{\prime} w^{\prime}\right) w^{* \prime \prime} \\
& \left.+\left(4 w^{\prime \prime} w^{\prime \prime \prime}+\frac{4}{r} w^{\prime \prime} w^{\prime \prime}+\frac{5}{r} w^{\prime} w^{\prime \prime \prime}-\frac{5}{r^{2}} w^{\prime} w^{\prime \prime}-\frac{8}{r^{3}} w^{\prime} w^{\prime}\right) w^{* \prime}\right\}=\frac{3}{16} r^{2}
\end{aligned}
$$


which serves as the basic differential equation of a circular plate under uniform load for the plastic flow theory.

The corresponding basic differential equation for the plastic deformation theory of a circular plate under uniform load can be derived in a similar manner; one obtains

$$
\begin{aligned}
r w^{\prime \prime \prime} & +w^{\prime \prime}-\frac{1}{r} w^{\prime}-\frac{c_{1}}{5}\left\{6 r w^{\prime \prime} w^{\prime \prime} w^{\prime \prime \prime}+6 w^{\prime} w^{\prime \prime} w^{\prime \prime \prime}+\frac{3}{r} w^{\prime} w^{\prime} w^{\prime \prime \prime}\right. \\
& \left.+4 w^{\prime \prime} w^{\prime \prime} w^{\prime \prime}+\frac{3}{r} w^{\prime} w^{\prime \prime} w^{\prime \prime}-\frac{3}{r^{2}} w^{\prime} w^{\prime} w^{\prime \prime}-\frac{4}{r^{3}} w^{\prime} w^{\prime} w^{\prime}\right\}=\frac{3}{16} l_{0} r^{2}
\end{aligned}
$$

The problem of solving the non-linear differential equations (4.4) and (4.5) for $w\left(r, l_{0}\right)$ presents great mathematical difficulties, and there is little hope for obtaining solutions in closed form. We therefore will attempt to approximate the solutions by means of series of the following type

$$
w\left(r, l_{0}\right)=w_{1}(r) l_{0}+w_{3}(r) l_{0}^{3}+w_{5}(r) l_{0}^{5}+\cdots,
$$

where the coefficients $w_{1}, w_{3}, w_{5}$, etc. of the odd powers of the parameter $l_{0}$ depend on $r$ alone. Substituting this expansion into Eq. (4.4), and collecting terms involving like powers in $l_{0}$, we obtain a sequence of differential equations in the unknown functions $w_{1}, w_{3}, w_{5}, \cdots$. The first of these is

or

$$
\begin{aligned}
& r w_{1}^{\prime \prime \prime}+w_{1}^{\prime \prime}-\frac{1}{r} w_{1}^{\prime}=\frac{3}{16} r^{2} \\
& r \frac{d}{d r}\left[\frac{1}{r} \frac{d}{d r}\left(r \frac{d w_{1}}{d r}\right)\right]=\frac{3}{16} r^{2}
\end{aligned}
$$

the next contains $w_{1}$ and $w_{3}$; only $w_{3}$ is unknown, however, since $w_{1}$ can be determined from Eq. (4.7). This procedure can be continued until the desired number of coefficients in (4.6) are found.

In view of the boundary conditions for a built-in circular plate,

$$
w\left(1, l_{0}\right)=0, \quad w^{\prime}\left(1, l_{0}\right)=0,
$$

and the requirement that $w\left(0, l_{0}\right)$ be finite, the solution of Eq. (4.7) is found to be

$$
w_{1}=\frac{3}{512}\left(r^{2}-1\right)^{2} ;
$$

this is nothing but the elastic solution. The differential equation for $w_{3}(r)$ then becomes

$$
r \frac{d}{d r}\left[\frac{1}{r} \frac{d}{d r}\left(r \frac{d w_{3}}{d r}\right)\right]=\frac{72 b_{1}}{5(128)^{3}}\left\{143 r^{6}-129 r^{4}+30 r^{2}\right\}
$$

and that for $w_{5}$

$$
\begin{aligned}
r \frac{d}{d r}\left[\frac{1}{r} \frac{d}{d r}\left(r \frac{d w_{5}}{d r}\right)\right]=\frac{8 \cdot 3^{5} \cdot 10^{11}}{25(128)^{7}}\left\{350,493 r^{10}-622,668 r^{8}\right. \\
\left.+419,112 r^{6}-128,067 r^{4}+15,270 r^{2}\right\} .
\end{aligned}
$$

Integrating these equations and substituting the results into the series (4.6), we obtain 


$$
\begin{aligned}
w\left(r, l_{0}\right)=\frac{3}{512} & \left(r^{2}-1\right)^{2} l_{0}+\frac{45}{4(12.8)^{4}}\left\{143 r^{8}-344 r^{6}+360 r^{4}\right. \\
& \left.-260 r^{2}+101\right\} l_{0}^{3}+\frac{324}{(12.8)^{7}}\left\{584,155 r^{12}-1,868,004 r^{10}\right. \\
& +2,619,450 r^{8}-2,134,450 r^{6}+1,145,250 r^{4} \\
& \left.-529,860 r^{2}+183,459\right\} l_{0}^{5}+\cdots
\end{aligned}
$$

This equation is the series development up to terms of the 5 th order in $l_{0}$ of the solution $w\left(r, l_{0}\right)$ to the differential equation (4.4) of the plastic flow theory.

Beginning with the differential Eq. (4.5), a similar expansion can be obtained for the deformation theory. The functions $w_{1}(r)$ and $w_{3}(r)$ in the series solution as given by the deformation theory are found to be identical with the corresponding functions obtained for the flow theory. This property can be shown to exist even under more general conditions; however, this fact will not be proved here. The first difference between the two theories occurs for the function $w_{5}$. Up to terms of this order, the series representation of the deflection furnished by the deformation theory is found to be:

$$
\begin{aligned}
w\left(r, l_{0}\right)=\frac{3}{512} & \left(r^{2}-1\right)^{2} l_{0}+\frac{45}{4(12.8)^{4}}\left\{143 r^{8}-344 r^{6}+360 r^{4}\right. \\
& \left.-260 r^{2}+101\right\} l_{0}^{3}+\frac{540}{(12.8)^{7}}\left\{352,495 r^{12}-1,130,796 r^{10}\right. \\
& +1,591,650 r^{8}-1,300,750 r^{6}+697,500 r^{4} \\
& \left.-320,340 r^{2}+110,241\right\} l_{0}^{5}+\cdots .
\end{aligned}
$$

The stress distribution throughout the circular plate can be calculated in the case of the plastic flow theory by transforming expression (3.4) into polar coordinates, substituting Eq. (4.12), and then integrating. The resulting non-dimensional expressions for the radial stress component $P$ and the circumferential stress component $Q$ together with those similarly obtained for the plastic deformation theory are valid for all values of $l_{0} \leq l_{0}^{\prime}$, where $l_{0}^{\prime}$ represents the maximum load which our assumed stress-strain law entitles us to consider. In order to calculate this load we return to Eq. (4.1). For uniaxial stress $l_{0}^{\prime}$ would correspond to the maximum height of the dashed curve in Fig. 1; for the combined state of stress we are considering, the stress-strain curve expresses the relation between the intensities of stress and strain, where the stress intensity

$$
T=3 / 2 r_{i} r_{i i},
$$

reduces for simple tension to $T=\rho^{2}$. We have already seen that $E=2 / 3 e_{i j} e_{i j}$ reduces to $\epsilon^{2}$ for simple tension. Thus, for combined states of stress, the coordinate axes of the dashed curve of Fig. 1 are labeled $T^{1 / 2}$ and $E^{1 / 2}$.

The non-dimensional stresis intensity $S=T / G_{0}^{2}=3 / 2 s_{i j} s_{i j}$ for the example being considered here is

$$
S=P^{2}+Q^{2}-P Q .
$$


Since the maximum value of $S^{1 / 2}$ occurs at the boundary, i.e., $x_{3}= \pm 1, r=1$, we find that for $l_{0}=l_{0}^{\prime}=.09$ a value $S^{1 / 2}=.01312$ is reached which takes us to the top of the stress-strain intensity curve in Fig. 1.

A review of the stress distribution on the upper surface of the plate shows that for $l_{0}=.02$, see Fig. 2, there exists no appreciable difference between the data as given

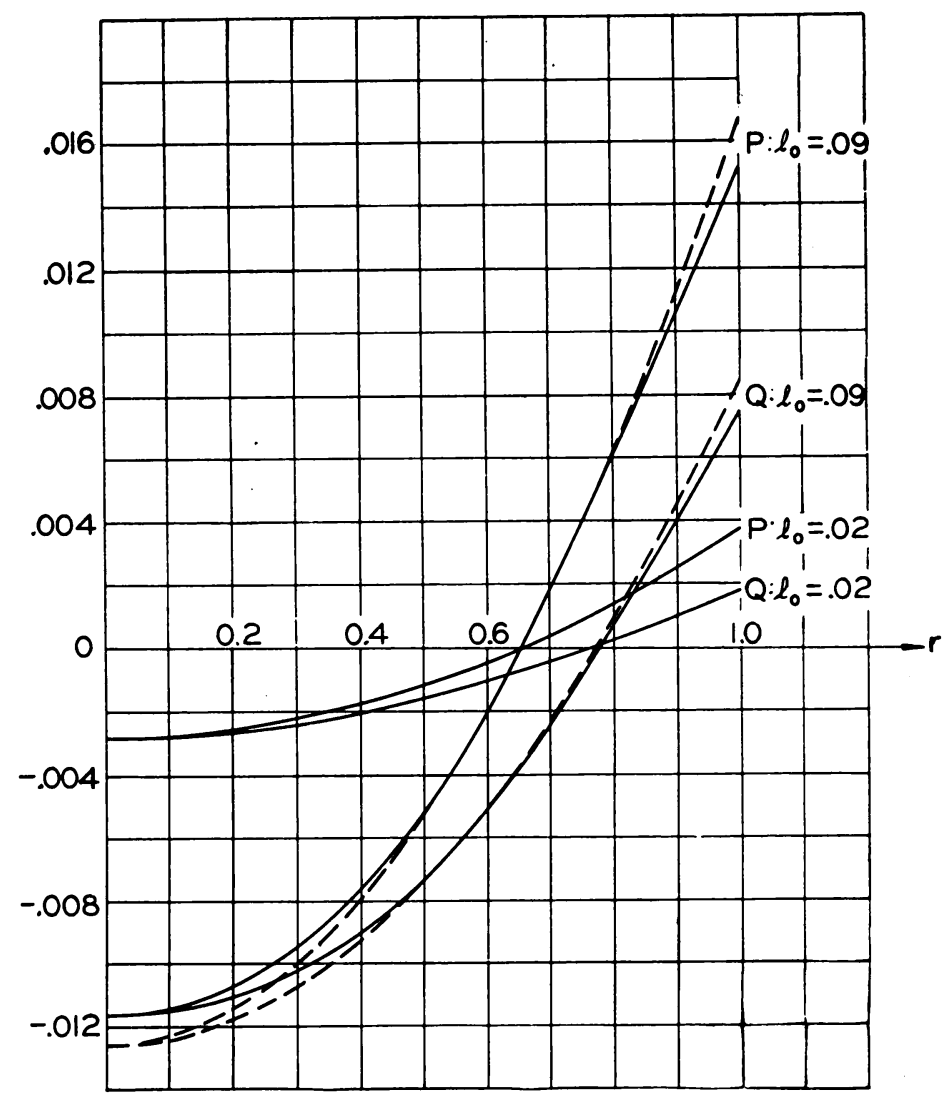

Fig. 2. Curves for the non-dimensional radial and circumferential stresses in the upper surface of the circular plate for $l_{0}=.02$ and $l_{0}=.09$ as given by the plastic theories (full curves) and the elastic theory (dashed curves).

by the elastic theory and the two plastic theories; to within five significant decimal places, the third order terms in $l_{0}$ have a negligible influence, and those of the fifth order have no influence at all. As the load increases to $l_{0}=.04$, there is a slight deviation which becomes more and more pronounced as $l_{0}$ increases; however, to within five decimal places there is at no time a difference between the values given by the theories of plastic flow and of plastic deformation. For $l_{0}=.06$ the third order terms have an influence ranging to $3.46 \%$ of the first order terms, while the fifth order terms reach only $.53 \%$ of the first order terms. Third order terms range to an influence of $7.8 \%$, and fifth order to $2.5 \%$ for the ultimate load $l_{0}=l_{0}^{\prime}=.09$. From this it is estimated that the solutions given by (4.12) and (4.13) approximate the real solutions with sufficient accuracy. 
In order to obtain the work $D$ done on the plate we calculate the following integral:

$$
\begin{aligned}
D & =2 \pi \int_{0}^{R_{0}} \int_{0}^{t} q_{0} \frac{\partial W}{\partial t} d t R d R \\
& =2 \pi \int_{0}^{R_{0}}\left[q_{0} W-\int_{0}^{t} W \frac{\partial q_{0}}{\partial t} d t\right] R d R .
\end{aligned}
$$

In non-dimensional form—see (3.2)—the above reduces to

$$
D=\pi G_{0} h R_{0}^{2} \int_{0}^{1}\left[l_{0} w-\int_{0}^{t} w \frac{\partial l_{0}}{\partial t} d t\right] r d r
$$

or by changing the time parameter again to $l_{0}$ we obtain

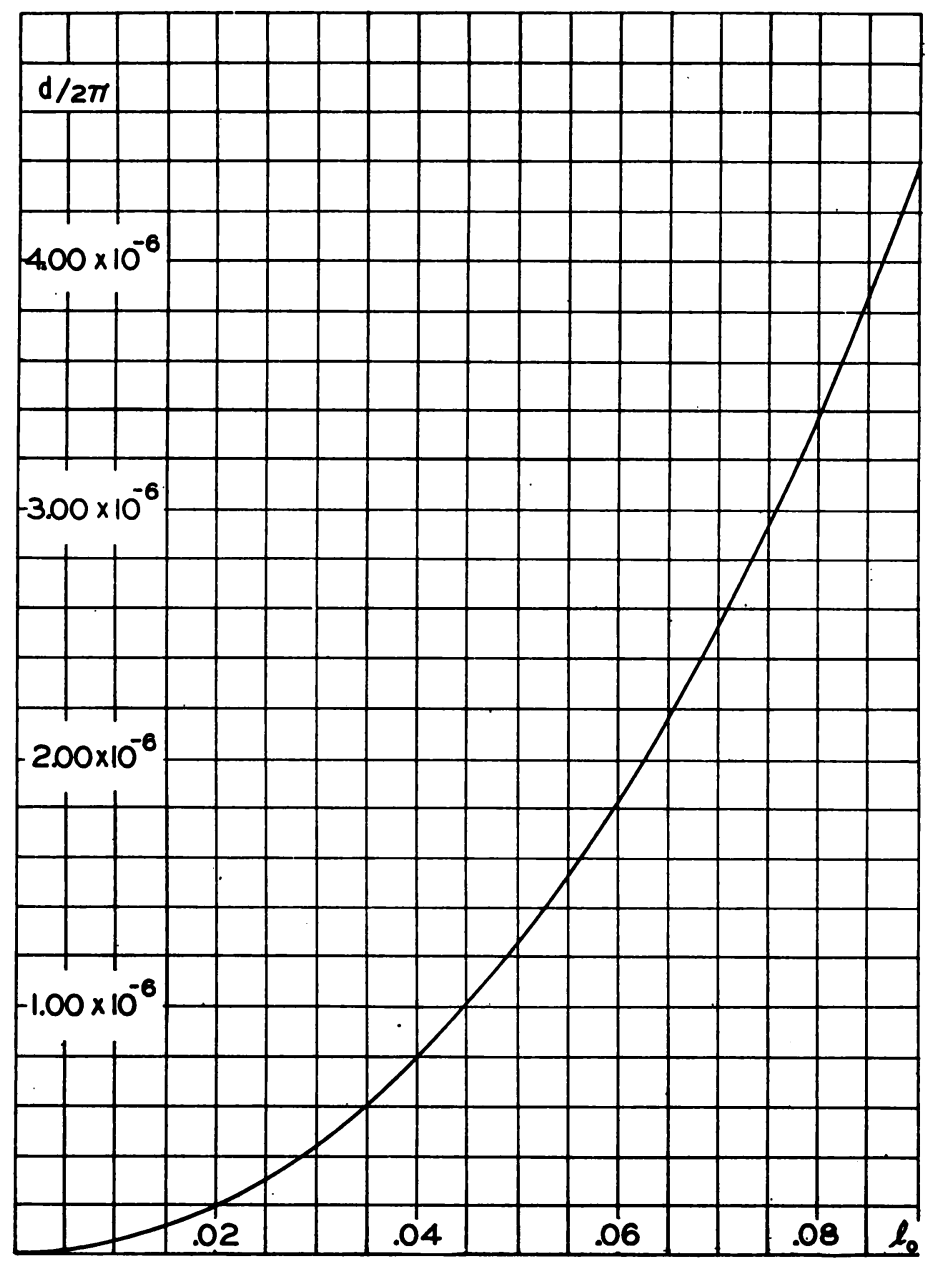

Fig. 3. Relation between non-dimensional loads $l_{0}$ versus non-dimensional work $d$ as given by Eq. (4.14). 


$$
D=\frac{1}{2} G_{0} h R_{0}^{2} d
$$

.. here the non-dimensional work $d$ is given by

$$
d=2 \pi \int_{0}^{1}\left[l_{0} w-\int_{0}^{l_{0}} w d l_{0}\right] r d r
$$

A graph of $d / 2 \pi$ versus $l_{0}$ is given in Fig. 3. We note that in the purely elastic case, where $w$ reduces to simply the first term of (4.12) or (4.13), the above formula (4.14) reduces to the familiar form

$$
d=\pi \int_{0}^{1} l_{0} w r d r
$$

In conclusion we can state that though the theories of plastic flow and plastic deformation are founded on different hypotheses their predictions for circular plates under uniform loads and for the specific material considered here are identical for all practical purposes. Both plastic theories however, give a deviation from the elastic solution ranging to better than $10 \%$ depending on the load and the material point under consideration.

\section{Bibliography}

[1] A. A. Ilyushin, Relation between the Theory of St. Venant-Lévy-Mises and the Theory of Small Elastic-Plastic Deformations, Prikl. Mat. Mekh. 9, 207-218 (1945).

[2] W. Prager, On Isotropic Materials with Continuous Transition from Elastic to Plastic State, Proc.' 5th Internat. Congr. Appl. Mech., Cambridge (Mass.) 1938, pp. 234-237.

[3] W. Prager, A New Mathematical Theory of Plasticity, Prikl. Mat. Mekh. 5, 419-428 (1941).

[4] G. H. Handelman, C. C. Lin, and W. Prager, On the Mechanical Behaviour of Metals in the StrainHardening Range, Q. Appl. Math. 4, 397-407 (1947).

[5] L. Prandtl, Spannungsverteilung in plastischen Körpern, Proc. 1st Internat. Congr. Appl. Mech., Delft 1924, pp. 43-54.

[6] E. Reuss, Berücksichtigung der elastischen Formaenderungen in der Plastizitaetstheorie, Z. angew. Math. Mech. 10, 266-274 (1930).

[7] H. Hencky, Zur Theorie plastischer Deformationen und der hierdurch im Material hervorgerufenen Nachspannungen, Z. angew. Math. Mech. 4, 323-334 (1924).

[8] A. Nadai, Plasticity, a Mechanics of the Plastic State of Matter, McGraw-Hill Book Co., Inc., New York, 1931.

[9] W. Prager, Strain-Hardening under Combined Stresses, J. Appl. Phys. 16, 837-840 (1945).

[10] A. A. Ilyushin, Some Problems in the Theory of Plastic Deformations, Prikl. Mat. Mekh. 7, 245-272 (1943).

[11] V. V. Sokolovsky, Elastic-Plastic Bending of Circular and Annular Plates, Prikl. Mat. Mekh. 8, 141-166 (1944).

[12] I. S. Sokolnikoff, Mathematical Theory of Elasticity, mimeographed lecture notes, Brown Univ., Providence, 1941.

[13] S. Timoshenko, Plates and Shells, McGraw-Hill Book Co., Inc., New York, 1940. 